\title{
Amyloidosis and Cardiac Surgery
}

\author{
Pavel Zacek and Jan Harrer \\ Department of Cardiac Surgery, Charles University Prague, \\ Faculty of Medicine in Hradec Králové, \\ and University Hospital Hradec Králové \\ Czech Republic
}

\section{Introduction}

Cardiac amyloidosis is a rare and complex pathology resulting in infiltrative restrictive cardiomyopathy and there are only very few clinical pathways through which the diseased amyloidotic heart may be a subject of cardiosurgical intervention. Cardiac surgery may rarely be warranted in very selected cases, and, on the other hand, may be performed mistakenly in misdiagnosed patients with cardiac amyloidosis. In clinical terms, the accumulation of expertise is hampered by the scarcity of its occurrence, heterogeneity of etiological subgroups, late clinical manifestation and polymorphous nature of symptoms.

The term amylon was first coined by Matthias Schleiden, German botanist, in 1838, for waxy starch in plants. In 1842 Karl Rokitansky described amyloidosis in connection with hepatosplenomegaly and Rudolf Virchow used in 1854 iodine for staining of amyloid. Today, more than one and half century later, we recognize amyloidosis as a heterogeneous infiltrative disease in which insoluble protein deposits are accumulated in various organs with deleterious effects on their functional integrity. Kidney, heart, blood vessels, central and peripheral nervous systems, liver, intestines, lungs, eyes, skin or bones may be affected (Cohen 1967).

\section{Classification of cardiac amyloidosis}

Cardiac amyloidosis is classified with regard to the origin of protein precursors in 6 types: primary (AL), secondary (reactive, AA), senile systemic, hereditary (familial), isolated atrial and hemodialysis-associated amyloidosis. Differentiation between these forms is based on immunohistochemical and genetic testing and implies the patient's prognosis and therapeutic strategies.

Primary amyloidosis (AL) is the disease of immunoglobulin light-chain proteins produced from plasma cells in multiple myeloma and other plasma cell dyscrasias (Waldenström macroglobulinemia, B-cell lymphoma, a. o.). Its incidence is rare, estimated for 8.9 per million population (Kyle, Linos et al. 1992), however, its clinical course is aggressive with poor prognosis. The median survival without treatment is 13 months and may be prolonged to 17 months with melphalane and prednisone therapy (Kyle, Gertz et al. 1997). Predominant cardiac involvement is the cause of dim course with median life expectancy of 4 months since clinical manifestation of congestive heart failure. Death is attributed to 
intractable low cardiac output or fatal arrhytmia. Sudden cardiac death may be preceded by syncope (Chamarthi, Dubrey et al. 1997).

Secondary amyloidosis (AA) is caused by the accumulation of amyloid A fibrils formed from an acute-phase reactant, serum amyloid A protein, in chronic inflammatory diseases like rheumatoid arthritis, familial Mediterranean fever, chronic infections, chronic lung diseases, tuberculosis, and inflammatory bowel disease. The heart seems to be affected less frequently whereas the kidney involvement leads to proteinuria and renal failure. The treatment of the underlying inflammatory process can reverse the disease (Gillmore, Lovat et al. 2001).

Senile systemic amyloidosis is related to liver production of a wild-type transthyretin transport protein (TTR). Amyloid deposits can be found in the heart, aorta, brain, pancreas, lung, liver, kidneys, and other organs. Senile systemic amyloidosis seems to be an age related disease, affecting predominantly men above the age of 70 , with age-increasing incidence. Median survival of 75 months indicates less aggressive course of the disease (Cohen 1967; Ng, Connors et al. 2005).

Hereditary (familial) amyloidosis is an autosomal dominant disease in which genetically mutated proteins, namely TTR, form the insoluble deposits. More than 80 transthyretin mutation have been identified as well as mutations in other proteins (fibrinogen Aa, lysozyme, apolipoprotein A-I, and gelsolin) have also been reported (Connors, Richardson et al. 2000). Besides cardiac involvement the other manifestations are peripheral and autonomic polyneuropathy with mainly gastrointestinal symptoms, renal impairment.

Isolated atrial amyloidosis (AANF) is caused by atrial natriuretic peptide secretion in response to atrial dilation in valvular disease and chronic atrial fibrillation as well as in correlation to increasing age. Thin atrial amyloid deposits however do not affect significantly the cardiac performance. Cardiac amyloidosis may also develop in patients receiving long-term dialysis due to accumulation of beta2-microglobulin from chronic uremia (Gorevic, Casey et al. 1985). Amyloid deposits may be found in myocardium, pericardium and valves with minimal clinical impact (Noel, Zingraff et al. 1987).

\section{Symptoms and diagnosis}

Early diagnosis of cardiac amyloidosis is not easy since its symptoms are polymorphous and do not clearly lead clinicians to think about a rare diagnosis. The dominant pathophysiology is restrictive cardiomyopathy resulting in a diastolic failure. Frequent are arrhythmias, conductions disorders and syncopes. Angina may be present from obstructive intraluminal coronary microangiopathy (Narang, Chopra et al. 1993; Whitaker, Tungekar et al. 2004; Neben-Wittich, Wittich et al. 2005; Tsai, Seldin et al. 2011) which, in usual absence of epicardial coronary stenoses, can be classified as syndrome $\mathrm{X}$ - (Yagishita, Tanimoto et al. 2009). Low-voltage QRS amplitudes on ECG $(\leq 10 \mathrm{mV}$ in all precordial leads or $\leq 5 \mathrm{mV}$ in all limb leads) is a relatively constant finding but not very specific since it may be present also in obesity, emphysema, effusion, hypothyroidism and other clinical conditions (Shah, Inoue et al. 2006). Atrial fibrillation is common.

Besides some indicative information from patient's history (hematological disorders, chronic inflammatory processes, polyneuropathy) echocardiography, in current clinical practice, has the potential to raise suspicion on cardiac amyloidosis in a given patient. Echocardiography diagnosis of cardiac amyloidosis is based on combination of two dimensional (2D) a Doppler image. In 2D both left and right ventricular wall thickness is 
increased. Size of the ventricles remains unchanged while the atria are dilated. Myocardium displays highly abnormal texture described as "granular and sparkling" appearance due to acoustic mismatch between highly reflective amyloid deposits and normal myocardial tissue (Siqueira-Filho, Cunha et al. 1981). Pericardial effusion and signs of pulmonary hypertension are common. Pulsed wave Doppler parameters show diastolic left ventricular dysfunction, typically restrictive pattern, i.e. increased velocity of passive LV filling transmitral E wave and shortening of its deceleration time, shortening of isovolumic relaxation time and inversion of systolic and diastolic pulmonary vein velocity ratio.

Cardiac catheterization can confirm the nonspecific pathophysiology of restrictive cardiomyopathy (elevation of diastolic pressure in both ventricles and right-sided pressure curve with a dip and plateau or square root sign). Normal coronarography despite angina complaints fits the diagnosis of cardiac amyloidosis. Cardiac magnetic resonance imaging enables to visualize in 3D and high-resolution morphologic dimensions of the heart and regional wall motion. Decreased tissue signal intensity along with late subendocardial tissue enhancement by gadolinium can be helpful in differentiating amyloid cardiomyopathy (Maceira, Joshi et al. 2005; Bucciarelli-Ducci, Locca et al. 2007).

Ultimate diagnostic tool, though employed at advanced stage of diagnosis workup, is the biopsy specimen with positive Congo red staining for amyloid. Endomyocardial biopsy, if positive in four samples, gives almost $100 \%$ diagnostic sensitivity for amyloidosis. Tissue specimen can also be obtained from rectal submucosa or by abdominal fat aspiration (with sensitivity ranging between $75-85 \%$, and $84-88 \%$, respectively (Shah, Inoue et al. 2006).

Diagnostic difficulties are obvious: before enough clinical findings are gathered to justify the use of sophisticated and invasive diagnostic tools, the pathway to correct diagnosis may be tedious. Incorporating the possibility of cardiac amyloidosis into clinician's thinking and careful consideration of all available data and findings is mandatory for obtaining the proper diagnosis fast.

\section{Non-surgical treatment}

Conservative management of restrictive cardiomyopathy resulting from structural myocardial alteration is limited. Diuretics are used to balance the signs of congestive heart failure, with narrow margin against low-cardiac output. Calcium channel blockers are contraindicated for negative inotropic effect, as well as beta-blockers (Griffiths, Hughes et al. 1982; Gertz, Falk et al. 1985; Gertz, Skinner et al. 1985). Administration of digoxin is risky because of its binding to amyloid fibrils and resulting toxic effects (Rubinow, Skinner et al. 1981). Implantation of permanent pacemaker may be necessitated for conduction disorders, with potential for alleviation of symptoms (Mathew, Olson et al. 1997).

Chemotherapy in AL amyloidosis, based on hematooncology strategy in treatment of multiple myeloma, includes administration of melphalan orally (with prednisone (Skinner, Anderson et al. 1996)) or in dose-intensive intravenous protocol, followed by autologous blood stem cell transplantation (Comenzo, Vosburgh et al. 1998; Moreau, Leblond et al. 1998). The rationale is to reduce or abolish the supply of amyloidogenic monoclonal light chain protein from the plasma cell clone which may facilitate the regression of plasma deposits and improve the quality of life. Alternatively, thalidomide with dexamethasone, or bortezomib may be administered (Charaf, Iskandar et al. 2009). In selected patients, the combined chemotherapy and stem cell transplantation may prolong the survival, however, it carries the risk of increased morbidity and mortality (Saba, Sutton et al. 1999). 


\section{Cardiac surgery in cardiac amyloidosis}

Cardiac surgery, in logical consequence of low prevalence of cardiac amyloidosis, difficult straightforward diagnostics and very limited scope of action due to the nature of the disease, has a relatively small window of experience. Majority of the authors have published anecdotic case reports and only data on transplanted patients accumulate the evidence from small cohorts. Basically, there are three clinical pathways in which a patient with cardiac amyloidosis may be referred for cardiac surgery:

\subsection{Amyloidosis misdiagnosed as a coronary artery or heart valve disease}

Unsuspected cardiac amyloidosis as a cause of sudden fatal circulatory collapse in the course other cardiological or surgical intervention has been reported rather early (Goldman and Legnami 1966; Lindholm and Wick 1986; Kotani, Hashimoto et al. 2000; Wang and Pollard 2000). Postmortem examination in these case reports usually reveals surprisingly advanced stage of structural damage of the myocardium by amyloid deposits. Striking disparity between the gross morphological alteration and paucity of both clinical signs and diagnostic findings underlines the obvious difficulties of proper management strategies in cardiac amyloidosis.

Fatal response of the amyloidotic myocardium to the insult conveyed by anesthesia, general or cardiac surgery can be explained by concurrence of various pathophysiology mechanisms. Potential dysbalance of circulating volume in reaction to administration of anesthetic drugs cannot be adequately compensated in restrictive cardiomyopathy. Vicious circle is further potentiated by the myocardial inability to increase its contractile performance as well as by diffuse myocardial ischemia and susceptibility to arrhythmias. Standard treatment options, pharmacological support or counterpulsation, usually fail to resolve the circulatory shock.

In recent era of high volume cardiac surgery, the risk of referral of cardiac amyloidosis misdiagnosed for coronary or valve disease for cardiac surgery operation keeps to be present despite current improvements in diagnostic process. Obvious difficulties in proper diagnosis making of cardiac amyloidosis are combined with systemic bias of routine clinical thinking. Patient's symptoms that are indicative of far more prevalent diagnoses, namely coronary artery disease, prompt to perform coronarography. Though not typical for cardiac amyloidosis, stenoses of epicardial coronary arteries can either be clearly present on coronarography or at least stenoses of some degree may be assumed as a sufficient explanation of angina symptoms. Once labeled as patients with coronary artery disease a routine echocardiography is usually performed with focus on systolic ventricular function and presence of mitral regurgitation. When cardiac hypertrophy is not strikingly present diastolic dysfunction can be easily overlooked or not taken into account.

Fatal outcome of coronary artery bypass grafting in misdiagnosed patients with cardiac amyloidosis has been reported by several authors (Massoudy, Szabo et al. 2003; Massias, Vyssoulis et al. 2006; Zacek, Medilek et al. 2007). In our institutional records (unpublished data) there were four fatal cases of undiagnosed cardiac amyloidosis indicated for coronary surgery $(3 x)$ or mitral and tricuspid valve repair $(1 x)$ (Fig. 1). In 3 ischemic patients, however, only two had debatable coronary stenoses while the third had a severe left main stenosis. Echocardiography was undiagnostic in all three of them. Postmortem microscopy revealed advanced stage of cardiac amyloidosis also with documented obliterative deposition of amyloid in small coronary vessels (Fig 2). The echocardiography of the patient 


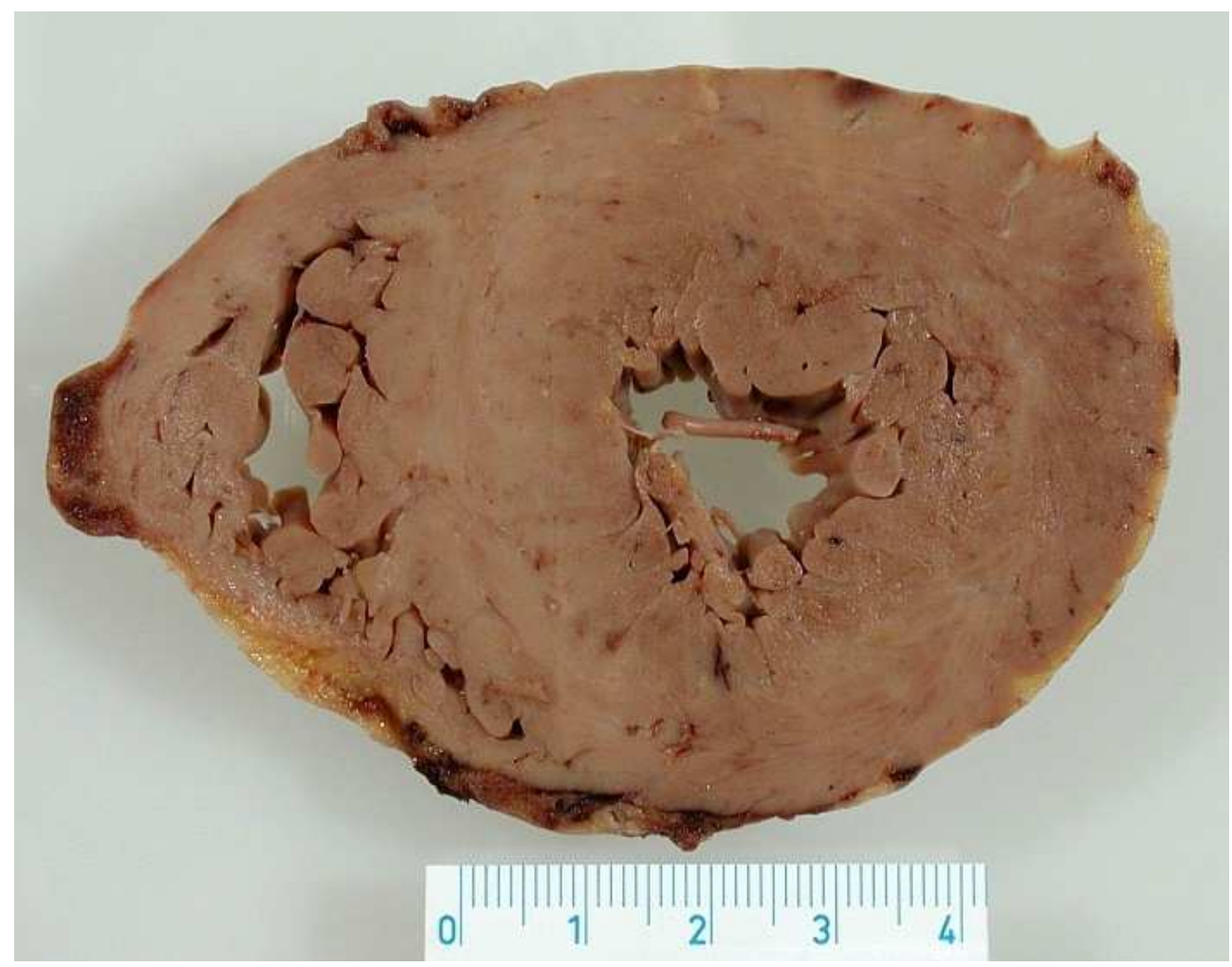

Fig. 1. Marked myocardial hypertrophy $610 \mathrm{~g}$ ) in 75-year-old patient indicated for coronary artery bypass grafting for hemodynamically significant left main stem stenosis. Type of cardiac amyloidosis not identified (negative for AL, AA and senile amyloidosis). Besides presyncope in patient history no relevant data indicative of possible presence of amyloidosis were traced.

indicated for mitral and tricuspid surgery displayed no clear indications of amyloid restrictive cardiomyopathy even on retrospective reevaluation.

From the published data there is evident lack of constant and specific signs that could reroute in real world the misdiagnosis in process and avoid disastrous and unjustified cardiac operation. Low QRS voltage seems to be very constant but of low specificity. Interestingly, constant is the surgeon's immediate tactile recognition of rubbery, stiff and nodular surface of myocardium. In obvious absence of diagnostic pattern that can safely indicate the correct diagnosis of cardiac amyloidosis the only advice is, first, meticulously include in consideration all the available data from patient history and examination, and, second, be sensitive for "small discrepancies" between the symptoms and objective findings (e.g., two of our patients had no clear angina but an effort dyspnea and fatigue were taken as an equivalent of this in presence of moderate stenoses on coronarography).

Correct diagnosis of cardiac amyloidosis would prevent disastrous outcome of bypass surgery in case of insignificant involvement of epicardial coronary arteries. Contrary to this, coincidence of severe coronary disease coinciding with amyloid disease will necessitate 


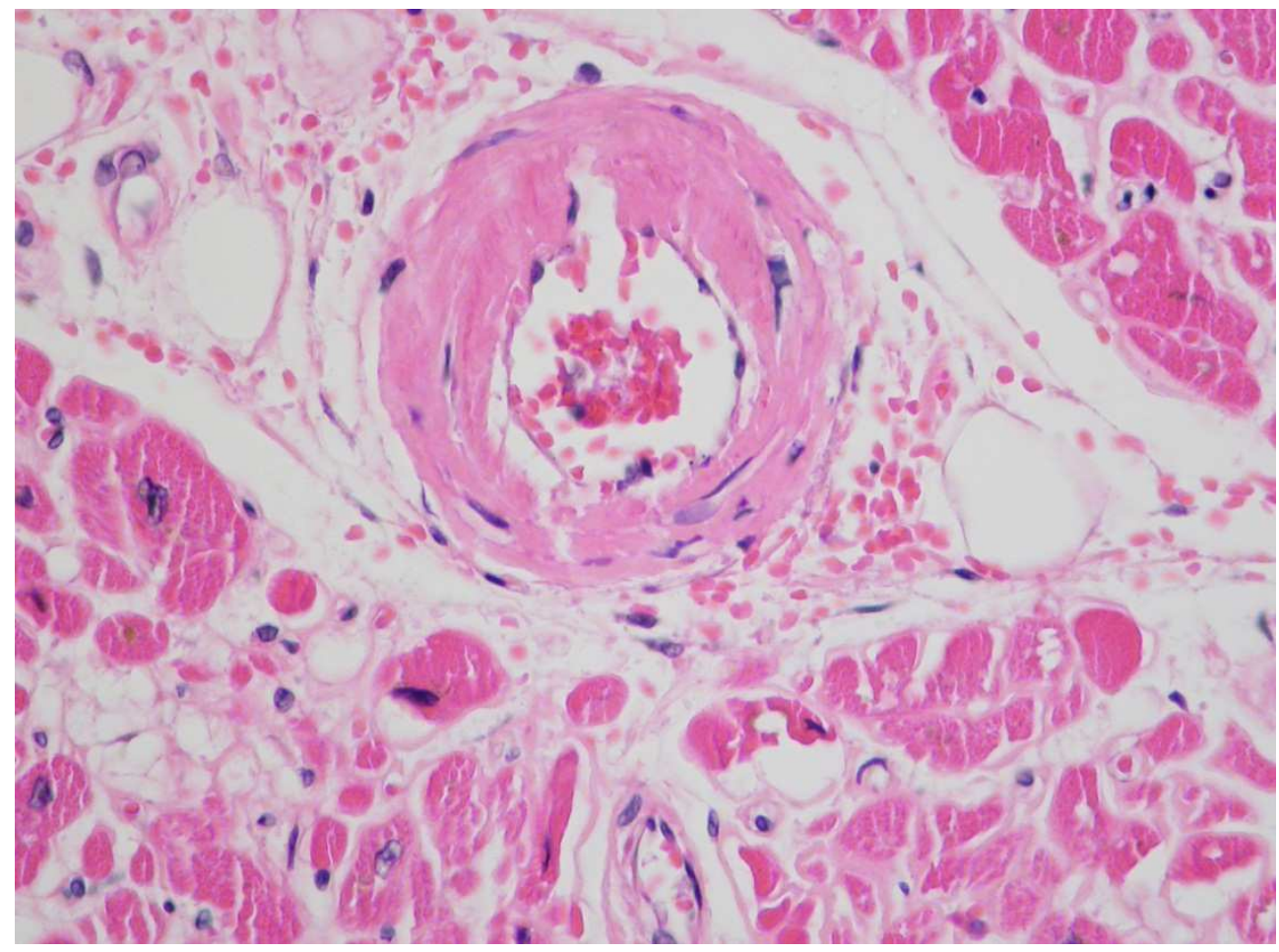

Fig. 2. Obstructive amyloid deposits in the wall of small intramyocardial artery (hematoxyllin-eosin, 400x)

percutaneous coronary intervention even in a difficult topography, instead of a surgery. Severe valve disease in a known cardiac amyloidosis would most probably lead to decision for conservative treatment.

\subsection{Planned surgical intervention in correctly diagnosed cardiac amyloidosis}

In contradiction to the abovementioned, very few reports describe intentional reconstructive cardiac surgery in patients with diagnosed cardiac amyloidosis. In 1983, Goffin coined a term dystrophic valvular amyloidosis for isolated amyloid deposits in cardiac valves (Goffin 1980). Successful aortic valve replacement was described by Iqbal (Iqbal, Reehana et al. 2006). In two cases, severe mitral regurgitation from papillary muscle or chordae rupture was successfully treated by valve repair and valve replacement (Coisne, Corbi et al. 2003; Nishi, Mitsuno et al. 2008). Obstructive intramural coronary amyloidosis was the speculative explanation for ischemic papillary muscle rupture in the latter case (Coisne, Corbi et al. 2003). Namai replaced successfully both mitral and aortic valve for endocarditis in a 62-year-old patient with multiple myeloma combined with renal amyloidosis (Namai, Sakurai et al. 2010). Uneventful postoperative course in these rare cases indicates that degree and distribution of morphological derangement, and moreover, the resulting functional deficit may vary considerably and therefore cannot be easily estimated prospectively. 


\subsection{Heart transplantation as ultimate surgical option in amyloid cardiomyopathy}

Cardiac transplantation is logically the only substantial surgical treatment option for the heart with profound structural alteration due to the vast deposits of amyloid. From methodological point of view, however, principal concerns involve the operative risk of transplant surgery in patients with multiorgan amyloid disease, and the subsequent risk of recurrent amyloidosis in the transplanted graft. The shortage of donor organs is also difficult to ignore.

The first reports of successful heart transplantation for cardiac amyloidosis are dated back to early 80-ties (Conner, Hosenpud et al. 1988; Hall and Hawkins 1994). Mc Gregor reported in 1998 the Mayo Clinic experience (McGregor, Rodeheffer et al. 1998) of 8 patients but the largest cohort was published by Dubrey in 2004 (Dubrey, Burke et al. 2001; Dubrey, Burke et al. 2004) comprising 24 patients transplanted over the period of 18 years. In AL amyloidosis patients, the survival was $50 \%, 50 \%$, and $20 \%$ at 1,2 , and 5 years in those without subsequent chemotherapy contrary to $71 \%, 71 \%$, and $36 \%$ respectively in 7 AL transplanted patients with additional chemotherapy. Without chemotherapy the median of amyloid recurrence in the graft was 11 months. Survival of the 7 non-AL amyloidosis patients was $86 \%, 86 \%$, and $64 \%$ at 1,2 , and 5 years. Overall 5-year survival of all amyloid patients was $38 \%$ in contrast to $67 \%$ in patients undergoing heart transplantation for other indications (Dubrey, Burke et al. 2004).

Regardless of the relatively small patient cohorts it can be assumed that the outcome of heart transplantation for AL amyloidosis is significantly worse than in general heart transplant population, namely due to progression of the systemic disease (Shah, Inoue et al. 2006; Luo, Chou et al. 2010).

In some clinical settings, heart transplantation may need to be combined with liver or kidney transplantation (Gillmore, Stangou et al. 2001; Schwartz, Kuiper et al. 2007; Audard, Matignon et al. 2009; Baumgratz, Vila et al. 2009). Liver transplantation should be instrumental or potentially curative in familial amyloidosis for abolishment of the aberrant transthyretin production. Scarcity of reported cases preclude consistent conclusion but it seems that precise knowledge of specific transthyretin mutation subtype may help to differentiate the outcome of transplantation strategy (Sharma, Perri et al. 2003).

\section{Conclusion}

In conclusion, cardiac amyloidosis is a complex pathology with poor prognosis since the onset of clinical manifestation even at the dawn of modern chemotherapy and stem cell transplantation. Should this disease be addressed by cardiac surgery the only therapeutic option is heart transplantation which, however, still remains to be debatable in view of postoperative results, recurrent amyloid disease of the graft and donor-organ shortage. Intentional cardiosurgical procedure for accompanying cardiac disorders can hardly be advised even in selected patients. On contrary, maximal vigilance has to be maintained to avoid misdiagnosing cardiac surgery for other more frequent cardiac maladies which otherwise can lead to unnecessary operation with fatal outcome.

\section{References}

Audard, V., M. Matignon, et al. (2009). Successful long-term outcome of the first combined heart and kidney transplant in a patient with systemic $\mathrm{Al}$ amyloidosis. Am J Transplant Vol 9 No (1): 236-240. 
Baumgratz, J. F., J. H. Vila, et al. (2009). Heart transplantation in primary amyloidosis. Rev Bras Cir Cardiovasc Vol 24 No (3): 409-412.

Bucciarelli-Ducci, C., D. Locca, et al. (2007). Value of cardiovascular magnetic resonance for determining cardiac involvement in systemic amyloidosis. Eur Heart J Vol 28 No (10): 1186.

Cohen, A. S. (1967). Amyloidosis. N Engl J Med Vol 277 No (10): 522-530 contd.

Coisne, D., P. Corbi, et al. (2003). Obstructive intramural coronary amyloidosis and papillary muscle rupture. Heart Vol 89 No (2): E8.

Comenzo, R. L., E. Vosburgh, et al. (1998). Dose-intensive melphalan with blood stem-cell support for the treatment of AL (amyloid light-chain) amyloidosis: survival and responses in 25 patients. Blood Vol 91 No (10): 3662-3670.

Conner, R., J. D. Hosenpud, et al. (1988). Heart transplantation for cardiac amyloidosis: successful one-year outcome despite recurrence of the disease. J Heart Transplant Vol 7 No (2): 165-167.

Connors, L. H., A. M. Richardson, et al. (2000). Tabulation of transthyretin (TTR) variants as of 1/1/2000. Amyloid Vol 7 No (1): 54-69.

Dubrey, S. W., M. M. Burke, et al. (2004). Cardiac transplantation for amyloid heart disease: the United Kingdom experience. J Heart Lung Transplant Vol 23 No (10): 1142-1153.

Dubrey, S. W., M. M. Burke, et al. (2001). Long term results of heart transplantation in patients with amyloid heart disease. Heart Vol 85 No (2): 202-207.

Gertz, M. A., R. H. Falk, et al. (1985). Worsening of congestive heart failure in amyloid heart disease treated by calcium channel-blocking agents. Am J Cardiol Vol 55 No (13 Pt 1): 1645.

Gertz, M. A., M. Skinner, et al. (1985). Selective binding of nifedipine to amyloid fibrils. Am J Cardiol Vol 55 No (13 Pt 1): 1646.

Gillmore, J. D., L. B. Lovat, et al. (2001). Amyloid load and clinical outcome in AA amyloidosis in relation to circulating concentration of serum amyloid A protein. Lancet Vol 358 No (9275): 24-29.

Gillmore, J. D., A. J. Stangou, et al. (2001). Clinical and biochemical outcome of hepatorenal transplantation for hereditary systemic amyloidosis associated with apolipoprotein AI Gly26Arg. Transplantation Vol 71 No (7): 986-992.

Goffin, Y. (1980). Microscopic amyloid deposits in the heart valves: a common local complication of chronic damage and scarring. J Clin Pathol Vol 33 No (3): 262-268.

Goldman, B. S. and F. A. Legnami (1966). Senile cardiac amyloidosis: autopsy finding in a patient dying after implantation of a cardiac pacemaker. Can Med Assoc J Vol 94 No (20): 1055-1058.

Gorevic, P. D., T. T. Casey, et al. (1985). Beta-2 microglobulin is an amyloidogenic protein in man. J Clin Invest Vol 76 No (6): 2425-2429.

Griffiths, B. E., P. Hughes, et al. (1982). Cardiac amyloidosis with asymmetrical septal hypertrophy and deterioration after nifedipine. Thorax Vol 37 No (9): 711-712.

Hall, R. and P. N. Hawkins (1994). Cardiac transplantation for AL amyloidosis. BMJ Vol 309 No (6962): 1135-1137.

Chamarthi, B., S. W. Dubrey, et al. (1997). Features and prognosis of exertional syncope in light-chain associated AL cardiac amyloidosis. Am J Cardiol Vol 80 No (9): 12421245. 
Charaf, E., S. B. Iskandar, et al. (2009). Cardiac amyloidosis responding to bortezomib: case report and review of literature. Curr Cardiol Rev Vol 5 No (3): 228-236.

Iqbal, S., S. Reehana, et al. (2006). Unique type of isolated cardiac valvular amyloidosis. J Cardiothorac Surg Vol 1 No: 38.

Kotani, N., H. Hashimoto, et al. (2000). Fatal perioperative myocardial infarction in four patients with cardiac amyloidosis. Anesthesiology Vol 92 No (3): 873-875.

Kyle, R. A., M. A. Gertz, et al. (1997). A trial of three regimens for primary amyloidosis: colchicine alone, melphalan and prednisone, and melphalan, prednisone, and colchicine. N Engl J Med Vol 336 No (17): 1202-1207.

Kyle, R. A., A. Linos, et al. (1992). Incidence and natural history of primary systemic amyloidosis in Olmsted County, Minnesota, 1950 through 1989. Blood Vol 79 No (7): 1817-1822.

Lindholm, P. F. and M. R. Wick (1986). Isolated cardiac amyloidosis associated with sudden death. Arch Pathol Lab Med Vol 110 No (3): 243-245.

Luo, J. M., N. K. Chou, et al. (2010). Heart transplantation in patients with amyloidosis. Transplant Proc Vol 42 No (3): 927-929.

Maceira, A. M., J. Joshi, et al. (2005). Cardiovascular magnetic resonance in cardiac amyloidosis. Circulation Vol 111 No (2): 186-193.

Massias, S., G. Vyssoulis, et al. (2006). Progressive heart failure in a patient after coronary artery bypass grafting. Hellenic J Cardiol Vol 47 No (2): 114-117.

Massoudy, P., A. K. Szabo, et al. (2003). Amyloid of heart and lungs in a patient with low output syndrome after coronary artery bypass grafting. Herz Vol 28 No (5): 453-456.

Mathew, V., L. J. Olson, et al. (1997). Symptomatic conduction system disease in cardiac amyloidosis. Am J Cardiol Vol 80 No (11): 1491-1492.

McGregor, C., R. Rodeheffer, et al. (1998). Heart transplantation in primary systemic amyloidosis. J Heart Lung Transplant Vol 17 No: 51.

Moreau, P., V. Leblond, et al. (1998). Prognostic factors for survival and response after highdose therapy and autologous stem cell transplantation in systemic AL amyloidosis: a report on 21 patients. Br J Haematol Vol 101 No (4): 766-769.

Namai, A., M. Sakurai, et al. (2010). Cardiac surgery in a patient with multiple myeloma combined with renal amyloidosis. Gen Thorac Cardiovasc Surg Vol 58 No (7): 341343.

Narang, R., P. Chopra, et al. (1993). Cardiac amyloidosis presenting as ischemic heart disease. A case report and review of literature. Cardiology Vol 82 No (4): 294-300.

Neben-Wittich, M. A., C. M. Wittich, et al. (2005). Obstructive intramural coronary amyloidosis and myocardial ischemia are common in primary amyloidosis. Am J Med Vol 118 No (11): 1287.

$\mathrm{Ng}$, B., L. H. Connors, et al. (2005). Senile systemic amyloidosis presenting with heart failure: a comparison with light chain-associated amyloidosis. Arch Intern Med Vol 165 No (12): 1425-1429.

Nishi, H., M. Mitsuno, et al. (2008). Severe mitral regurgitation due to cardiac amyloidosis--a rare reason for ruptured chordae. Interact Cardiovasc Thorac Surg Vol 7 No (6): 11991200.

Noel, L. H., J. Zingraff, et al. (1987). Tissue distribution of dialysis amyloidosis. Clin Nephrol Vol 27 No (4): 175-178. 
Rubinow, A., M. Skinner, et al. (1981). Digoxin sensitivity in amyloid cardiomyopathy. Circulation Vol 63 No (6): 1285-1288.

Saba, N., D. Sutton, et al. (1999). High treatment-related mortality in cardiac amyloid patients undergoing autologous stem cell transplant. Bone Marrow Transplant Vol 24 No (8): 853-855.

Shah, K. B., Y. Inoue, et al. (2006). Amyloidosis and the heart: a comprehensive review. Arch Intern Med Vol 166 No (17): 1805-1813.

Sharma, P., R. E. Perri, et al. (2003). Outcome of liver transplantation for familial amyloidotic polyneuropathy. Liver Transpl Vol 9 No (12): 1273-1280.

Schwartz, B. G., J. J. Kuiper, et al. (2007). Combined cardiac and liver transplantation for the treatment of familial amyloidosis. Proc (Bayl Univ Med Cent) Vol 20 No (2): 146-148.

Siqueira-Filho, A. G., C. L. Cunha, et al. (1981). M-mode and two-dimensional echocardiographic features in cardiac amyloidosis. Circulation Vol 63 No (1): 188196.

Skinner, M., J. Anderson, et al. (1996). Treatment of 100 patients with primary amyloidosis: a randomized trial of melphalan, prednisone, and colchicine versus colchicine only. Am J Med Vol 100 No (3): 290-298.

Tsai, S. B., D. C. Seldin, et al. (2011). Myocardial infarction with "clean coronaries" caused by amyloid light-chain AL amyloidosis: a case report and literature review. Amyloid No.

Wang, M. M. and J. B. Pollard (2000). Postoperative ventricular fibrillation and undiagnosed primary amyloidosis. Anesthesiology Vol 92 No (3): 871-872.

Whitaker, D. C., M. F. Tungekar, et al. (2004). Angina with a normal coronary angiogram caused by amyloidosis. Heart Vol 90 No (9): e54.

Yagishita, A., S. Tanimoto, et al. (2009). Cardiac amyloidosis presumptively diagnosed as cardiac syndrome X. Circ J Vol 73 No (7): 1349-1351.

Zacek, P., K. Medilek, et al. (2007). Cardiac amyloidosis in the cardiosurgical operating room--a rare but fatal trap. Thorac Cardiovasc Surg Vol 55 No (2): 65-67. 


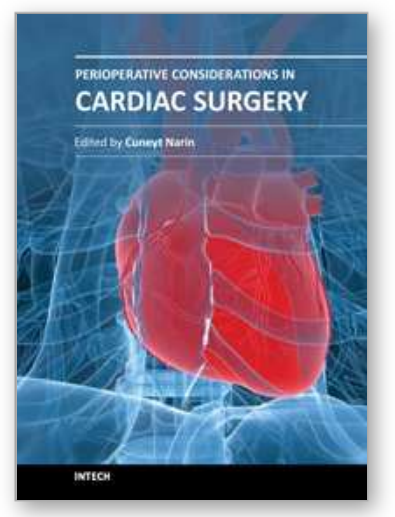

\author{
Perioperative Considerations in Cardiac Surgery \\ Edited by Prof. Cuneyt Narin
}

ISBN 978-953-51-0147-5

Hard cover, 378 pages

Publisher InTech

Published online 29, February, 2012

Published in print edition February, 2012

This book considers mainly the current perioperative care, as well as progresses in new cardiac surgery technologies. Perioperative strategies and new technologies in the field of cardiac surgery will continue to contribute to improvements in postoperative outcomes and enable the cardiac surgical society to optimize surgical procedures. This book should prove to be a useful reference for trainees, senior surgeons and nurses in cardiac surgery, as well as anesthesiologists, perfusionists, and all the related health care workers who are involved in taking care of patients with heart disease which require surgical therapy. I hope these internationally cumulative and diligent efforts will provide patients undergoing cardiac surgery with meticulous perioperative care methods.

\title{
How to reference
}

In order to correctly reference this scholarly work, feel free to copy and paste the following:

Pavel Zacek and Jan Harrer (2012). Amyloidosis and Cardiac Surgery, Perioperative Considerations in Cardiac Surgery, Prof. Cuneyt Narin (Ed.), ISBN: 978-953-51-0147-5, InTech, Available from: http://www.intechopen.com/books/perioperative-considerations-in-cardiac-surgery/cardiac-amyloidosis-versuscardiac-surgery

\section{INTECH}

open science | open minds

\section{InTech Europe}

University Campus STeP Ri Slavka Krautzeka 83/A 51000 Rijeka, Croatia Phone: +385 (51) 770447 Fax: +385 (51) 686166 www.intechopen.com

\section{InTech China}

Unit 405, Office Block, Hotel Equatorial Shanghai No.65, Yan An Road (West), Shanghai, 200040, China 中国上海市延安西路65号上海国际贵都大饭店办公楼405单元 Phone: +86-21-62489820

Fax: +86-21-62489821 
(C) 2012 The Author(s). Licensee IntechOpen. This is an open access article distributed under the terms of the Creative Commons Attribution 3.0 License, which permits unrestricted use, distribution, and reproduction in any medium, provided the original work is properly cited. 